\title{
Imagem pública da enfermeira: pesquisa documental (1910-1920)
}

\author{
Nurses' public image: documentary research (1910-1920) \\ Imagen pública de la enfermera: investigación documental (1910-1920) \\ Hugo Alberto Neves de Souza'; Keythluci Faria Trigueiro"; Alexandre Barbosa Oliveira"ll; \\ Margarida Bernardes ${ }^{\prime V}$; Antonio Marcos Tosoli Gomes ${ }^{v}$; Fernando Porto ${ }^{V I}$
}

\begin{abstract}
RESUMO
Objetivo: examinar os estudos desenvolvidos sobre a imagem pública da enfermeira brasileira veiculada em revistas ilustradas das décadas de 1910 e 1920, no Distrito Federal brasileiro. Método: realizada pesquisa documental, mediante análise historiográfica, com ênfase em publicações do início do século XX. Resultados: ratificaram a concorrência da enunciação da imagem pública da enfermeira nas circunstâncias da I Guerra Mundial, da Gripe Espanhola e também da Reforma Sanitária, liderada por Carlos Chagas, por meio dos efeitos simbólicos dos marcadores institucionais representados pelo uso do véu, gorro e touca. A liderança que prevaleceu na enunciação da imagem pública da enfermeira foi a investida pela Escola Prática de Enfermeiras da Cruz Vermelha Brasileira. Conclusão: direciona-se para além da concorrência entre as instituições de ensino, apontando vestígios para disputas em outro campo, o das relações internacionais.
\end{abstract}

Descritores: Enfermagem; história da enfermagem; historiografia; escolas de enfermagem.

\section{ABSTRACT}

Objective: to examine studies of Brazilian nurses' public image published in illustrated magazines in the 1910s and 1920s in Brazil's Federal District. Method: documentary research was conducted by historiographic analysis, with emphasis on early $20^{\text {th }}$ century publications. Results: the studies ratified the presence of competition in the enunciation of nursing's public image in the circumstances of World War I, the Spanish Flu and also the Sanitary Reform led by Carlos Chagas, through the symbolic effects of institutional markers represented by the use of the veil, nurse's cap and mob-cap. The leadership that prevailed in enunciating nurses' public image was the one invested in by the Brazilian Red Cross Nurses' Practical School. Conclusion: pointing beyond competition among educational institutions, this indicates traces of disputes in another field, international relations.

Descriptors: Nursing, history of nursing; historiography, schools nursing.

\section{RESUMEN}

Objetivo: examinar los estudios desarrollados sobre la imagen pública de la enfermera brasileña vehiculada en revistas ilustradas de las décadas de 1910 y 1920, en el Distrito Federal brasileño. Método: se realizó una investigación documental, mediante análisis historiográfico, con énfasis en publicaciones de principios del siglo XX. Resultados: ratificaron la competencia de la enunciación de la imagen pública de la enfermera en las circunstancias de la $1^{\text {a }}$ Guerra Mundial, de la Gripe Española y también de la Reforma Sanitaria, encabezada por Carlos Chagas, por medio de los efectos simbólicos de los marcadores institucionales representados por el uso del velo y gorro. El liderazgo que prevaleció en la enunciación de la imagen pública de la enfermera fue el de la Escuela Práctica de Enfermeras de la Cruz Roja Brasileña. Conclusión: se dirige más allá de la competencia entre las instituciones de enseñanza, señalando vestigios para disputas en otro campo, el de las relaciones internacionales.

Descriptores: Enfermería; história de la enfermeira; historiografia; facultades de enfermería.

\section{INTRODUÇÃO}

As publicações ilustradas da Revista da Semana e da Revista Fon-Fon foram duas concorrentes das mais lidas em tempos idos. A primeira foi lançada em 1900, a qual destacava imagens fotográficas com proposta de leitura do tipo leve, para mulheres ${ }^{1}$. A segunda, fundada em 1907, tratava de costumes e notícias do cotidiano, com destaque para propagandas comerciais e publicidades². Assim sendo, qual era a imagem pública da enfermeira no início do século XX veiculada nas revistas?

'Enfermeiro. Mestre em Enfermagem. Universidade Federal do Estado do Rio de Janeiro. Rio de Janeiro, Brasil. E-mail: hugoal.neves2@hotmail.com.

"Enfermeira. Universidade Federal do Estado do Rio de Janeiro. Brasil. E-mail: keythlucifaria@gmail.com.

I'Enfermeiro. Pós-doutorado. Professor Adjunto IV, Universidade Federal do Rio de Janeiro. Brasil. E-mail: alexbaroli@gmail.com.

IVEnfermeira. Pós-doutorado. Escola Superior de Guerra. Brasil. E-mail: margarbe@globo.com.

VEnfermeiro. Pós-doutorado. Professor Titular, Universidade do Estado do Rio de Janeiro. Brasil. E-mail: mtosoli@gmail.com.

VIEnfermeiro. Pós-doutorado. Professor Associado, Universidade Federal do Estado do Rio de Janeiro. E-mail: ramosporto@openlink.com.br. 
Foi possível identificar as representações características da marcação de atributos identitários das enfermeiras à época, tais como: o véu e o símbolo da cruz, na cor vermelha, oriundos da Escola e Cursos da Cruz Vermelha Brasileira; o véu e o símbolo da cruz, sem identificação de cor, do Curso da Policlínica de Botafogo; o gorro, com o símbolo da cruz na cor azul, da Escola Profissional de Enfermeiros e Enfermeiras e da Escola Profissional de Enfermeiras Alfredo Pinto e; a touca da Escola de Enfermeiras do Departamento Nacional de Saúde Pública ${ }^{3}$.

Evidenciou-se a concorrência entre as instituições de ensino, para enunciação da imagem pública da enfermeira brasileira, pela assinatura imagética relacionada aos atributos ostentados como composição da identidade institucional ${ }^{1}$.

Diante do exposto, objetivou-se examinar os estudos desenvolvidos sobre a imagem pública da enfermeira brasileira, por meio das revistas ilustradas das décadas de 1910 e 1920, no Distrito Federal brasileiro. Nesse sentido, buscou-se exercer olhar crítico sobre o conhecimento, ora produzido, acerca da história da enfermagem, tendo como foco de análise a veiculação da imagem da enfermeira nas primeiras décadas do século XX.

\section{REFERENCIAL TEÓRICO-METOdOLóGICO}

Trata-se de pesquisa documental de análise historiográfica, com ênfase em publicações do século XX, visando a construção da imagem pública da enfermeira para a história da enfermagem.

O termo historiografia ${ }^{4}$ foi utilizado para se discutir o que foi produzido sobre a imagem da enfermeira ${ }^{4}$. Com isto, vislumbrou-se a possibilidade de realizar o debate a partir do conhecimento produzido ao se reexaminar a construção da trama sociocultural.

Outro elemento teórico aplicado ao estudo foi o termo imagem. Este abrange dois domínios - mental e material - considerando que um não existe sem o outro. Isto implicou o processo de construção da imagem pública da enfermeira, para a sua idealização (mental), materializada para resultar a produção de sentido, o que conduziu à representação dessa profissional ${ }^{5}$.

A massa documental foi composta pela Revista da Semana e a Revista Fon-Fon das décadas de 1918 e 1929.

\section{RESULTADOS E DISCUSSÃo}

No bojo do contexto da I Guerra Mundial e das circunstâncias da epidemia da gripe espanhola, o discurso imagético era enunciado pela Cruz Vermelha Brasileira. À época, a instituição tomou para si o discurso de primeira instituição de ensino na formação de enfermeiras no Brasil, desconsiderando a criação da Escola Profissional de Enfermeiros e Enfermeiras, criada em 1890.

Na década de 1920, a defesa da imagem pública da enfermeira permaneceu por conta da Cruz Vermelha Brasileira. Assim, ela contou com a aliança da Escola de Enfermeiras do Departamento Nacional de Saúde Pública (1923), apesar desta última ter ressalvas para além da exclusão do masculino, ou seja, lutava para tomar para si o pioneirismo da formação. Logo, o que passou a estar em jogo foi o direito de enunciar a imagem da enfermeira de saúde pública como agente de implantação da enfermagem moderna no Distrito Federal.

No processo de inserção do Brasil na I Guerra Mundial, a mobilização de enfermeiras potencializou a presença delas nas páginas das revistas ilustradas. Sua veiculação pública retratava cenas de formação e atuação, com destaque para o atendimento aos feridos de guerra.

Diversos autores debruçaram-se em documentação daquela época e evidenciaram o destaque da Cruz Vermelha Brasileira, órgão central no Distrito Federal, na formação de enfermeiras para atuação na guerra. Em 1914, o Comitê das Damas da Cruz Vermelha Brasileira demandou a criação do Curso de Enfermeiras Voluntárias, justificada pela necessidade de prestação de cuidados em tempos de conflito e em calamidades públicas ${ }^{2,3,6-13}$.

Neste contexto, a Cruz Vermelha veiculou imagens das enfermeiras com o atributo do véu, em decorrência da representação plástica denominada The Greatest Mother in the World $(1918)^{14}$, que se refere a uma releitura da Pietá, de Michelangelo $(1498)^{1,15}$, como apresentada na Figura 1.

$\mathrm{Na}$ obra intitulada $\mathrm{O}$ livro do enfermeiro e da enfermeira - para o uso dos que se destinam à profissão de enfermagem e das pessoas que cuidam de doentes, de autoria de Getúlio dos Santos, secretário geral da Cruz vermelha Brasileira e diretor da instituição de ensino das enfermeiras, o médico criticou o surgimento de uma instituição de ensino criada, no alvorecer da República, para ambos os sexos, que não teria surtido o efeito esperado ${ }^{16}$.

Com base no discurso de Getúlio dos Santos sobre o efeito desejado, um estudo confiou em sua assertiva e a interpretou, talvez ingenuamente, como se ele tivesse, de fato, ignorado a criação da instituição de ensino, inaugurada em 1890, na argumentação de que se destinava à formação de enfermeiros ${ }^{13}$. 


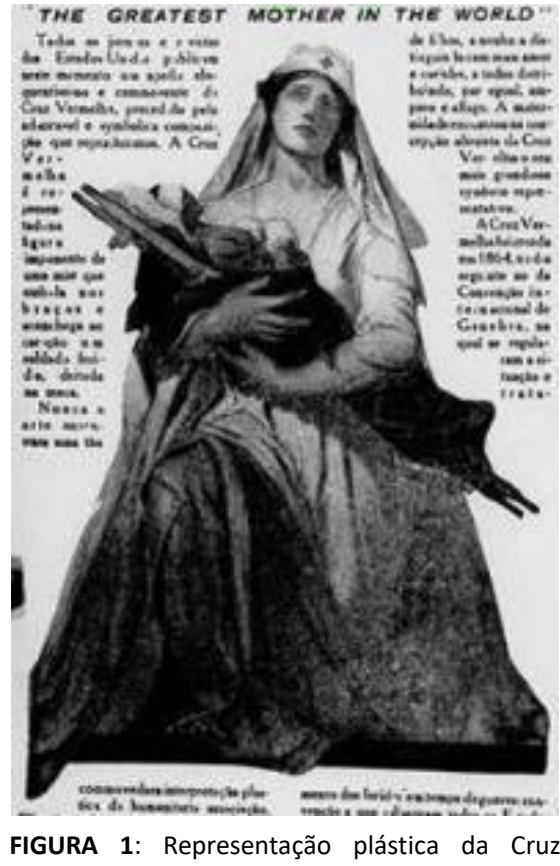

Vermelha. Rio de Janeiro, 1918.

Ao se examinar as argumentações do referido estudo ${ }^{13}$, identificaram-se algumas fragilidades ${ }^{13}$. Uma delas foi que a instituição de ensino não formava apenas enfermeiras, mas profissionais de ambos os sexos, como pode-se verificar na lista de formandos de 1906 da primeira turma ${ }^{17}$. A outra é referente à interpretação de Getúlio dos Santos sobre ter ignorado a criação de instituição anterior à Escola Prática da Cruz Vermelha Brasileira. Sobre isto, entende-se como discurso estratégico, pois, de fato, a única turma formada até 1921 foi aquela de 1906. Logo, o discurso de Getúlio dos Santos, em parte, retratava o que teria ocorrido à época sobre o efeito esperado, o que fez a pesquisadora, por falta de acesso a determinadas documentações, equivocar-se na construção de sua assertiva ${ }^{13}$.

Defender a enfermagem em prol do feminino era, ao mesmo tempo, defender as qualidades da mulher, o que, de certa forma, era um alvo da Cruz Vermelha Brasileira ${ }^{16}$. Para tanto, Getúlio dos Santos era um dos porta-vozes desse discurso, considerando a visibilidade e o poder simbólico da instituição que dirigia. Seu discurso sobre a preferência por mulheres, nessa atividade, pautava-se na sinceridade delas, bem como por serem mais comedidas do que os homens, destacando que elas necessitavam de formação para tornarem-se enfermeiras.

Ao seguir por esta vertente do discurso, vem à tona os congressos médicos latino-americanos ocorridos no período de 1904 a 1907. Neles, uma das discussões recaiu sobre a formação do enfermeiro e da enfermeira, com preferência para elas, ratificada pelas qualidades femininas ${ }^{18}$. Por outro lado, tem-se registro de que, desde o século XIX, na França, havia predomínio de enfermeiros nos hospitais franceses, sendo entendido que a feminização da profissão poderia colocar em risco o prestígio conquistado até aquele momento ${ }^{19}$, bem como nos hospitais dos Estados Unidos ${ }^{20,21}$, Holanda e Inglaterra ${ }^{22}$, o que foi inevitável com o desenvolvimento da enfermagem neste último país.

Cabe destacar que os resultados apontados para a exclusão do enfermeiro deveram-se ao investimento sistemático na imagem do feminino na profissão. Contudo, não significou a ausência deles na prática dos cuidados, como apontado em algumas pesquisas ${ }^{23,24}$.

A representação da imagem pública da enfermeira no término da I Guerra Mundial ${ }^{6}$ servia para a reprodução da crença simbólica na Cruz Vermelha Brasileira, conforme ilustra a Figura 2.

Foi possível identificar que se trata de cópia reproduzida, no Brasil, advinda da edição de maio da Motion Picture Magazine, periódico norte-americano que circulou entre 1911 e $1977^{19}$. Este dado torna-se relevante, pois o leitor de ontem e de hoje, ao ver a imagem da capa da Revista da Semana ${ }^{25}$, poderia pensar se tratar de enfermeira brasileira. A imagem veiculada conduz a pensar na apropriação de um modelo internacional para reforçar o que se tinha no Brasil.

A circunstância da publicação remete à assertiva de que a enfermagem precisava se (re)conhecer naquele contexto em função das necessidades de saúde que se apontavam, fosse nas bélicas, como na Guerra da Criméia, que evidenciou a figura de Florence Nightingale, ou na Guerra do Paraguai, que revelou a atuação da brasileira Anna Justina 
Ferreira Nery nas demandas da época voltadas para a saúde pública. Nesse jogo, as capas das revistas conferiam-Ihe visibilidade no campo social, o que contribuiu para o desenvolvimento da profissão ${ }^{26}$.

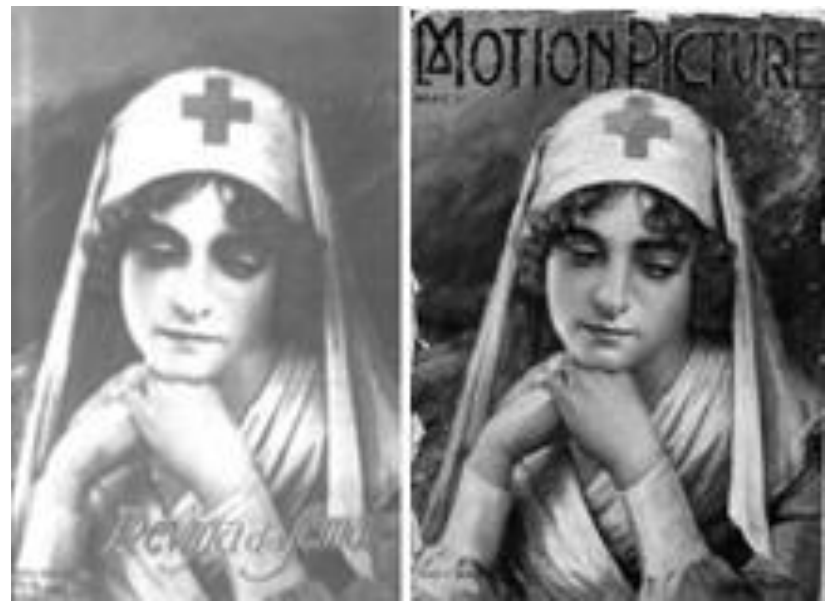

FIGURA 2: Capa da Revista da Semana. Rio de Janeiro, 1918.

Em meio às celebrações do fim da I Guerra Mundial, a Gripe Espanhola despontou como uma pandemia mais mortífera que o próprio conflito bélico. Nessa circunstância, a imagem pública da enfermeira foi reforçada com a atuação das instituições de ensino, com destaque para a Escola Prática de Enfermeiras da Cruz Vermelha Brasileira e para o Curso de Enfermeiras da Policlínica de Botafogo (1917-1920), no desenvolvimento de ações voltadas para a saúde pública, o que acabou deflagrando as condições para a criação do Departamento Nacional de Saúde Pública (1920), liderado por Carlos Chagas e, à reboque, da Escola de Enfermeiras do Departamento Nacional de Saúde Pública, subvencionada pela Fundação Rockefeller dois anos depois ${ }^{3,5,6,23}$, a qual daria origem à Escola de Enfermeiras Dona Anna Nery, em 1923.

Entraram em cena as enfermeiras norte-americanas, lideradas por Ethel Parsons, para promoverem a formação das que trabalhariam na área da saúde pública. Com isto, a criação desta escola também a inseriu em um campo de disputas onde, via imprensa ilustrada, buscava-se tomar para si o pioneirismo do ensino da enfermagem brasileira e a enunciação da imagem pública da enfermeira no país. Tal situação era, aparentemente, sustentada pelos efeitos da vitória dos Estados Unidos na I Guerra Mundial, como também era potencializada pelo subvencionamento da Fundação Rockefeller para a criação de mais uma escola para a formação de enfermeiras no Distrito Federal.

Alguns estudos, ao se pautarem na assertiva de ausência de instituições de formação de enfermeiras no país, adotaram como ponto de partida da profissionalização a criação da Escola do Departamento Nacional de Saúde Pública, em $1922^{27,28}$. Este dado, associado ao registro encontrado no relatório de Parsons de que não havia instituição de ensino para formar enfermeiras no Brasil, foi o argumento problematizador da pesquisa ${ }^{23}$, que se ateve à massa documental da Policlínica de Botafogo para refutar a ausência de escolas de enfermeiras à época. Esta instituição fez com que as pesquisadoras construíssem a assertiva de que o argumento de Ethel Parson não era só em virtude da concorrência sobre o pioneirismo entre as escolas, mas também para evitar o risco da enunciação da imagem da enfermeira por outra instituição de ensino.

Documentário datado de 1976, e reeditado em 2008, reconheceu a criação das instituições de ensino - Escola de Enfermagem Alfredo Pinto (1890) e da Cruz Vermelha Brasileira - Órgão Central (1914) -, mas registra que o Curso de Visitadoras da Cruz Vermelha Brasileira, datado de 1920, não teria surtido o efeito esperado em virtude da qualidade desejada pelos médicos do Departamento Nacional de Saúde Pública ${ }^{29}$. No debate historiográfico, tal afirmação foi problematizada, o que resultou em refutação ${ }^{30}$.

Isto possibilita a construção da assertiva de que não interessava ao Departamento Nacional de Saúde Pública, no Distrito Federal, obscurecer a imagem das enfermeiras norte-americanas, pela credibilidade que a instituição da Cruz Vermelha tinha no Rio de Janeiro, inclusive considerada como de utilidade pública, bem como pelo seu reconhecimento e representatividade internacional.

Descristalizar crenças dessa natureza não é tarefa fácil. Ethel Parsons era uma mulher de poder e prestígio na sociedade do Distrito Federal. Nesse sentido, apontar argumentos e contra-argumentos no âmbito de um debate historiográfico é também avançar na construção da História da enfermagem. 
Em 2011, pesquisa apresentada à comunidade acadêmica revelou a atuação de outra instituição de ensino na formação de enfermeiras, com a denominação de Curso de Parteiras-Enfermeiras da Pró-Matre, abrangendo o período 1928-1931 ${ }^{8}$ A datação certa do início da formação não foi possível detectar, mas infere-se que tenha ocorrido no final da década de 1910, tendo em vista a participação no atendimento aos acometidos pela Gripe Espanhola ${ }^{2}$.

Ao serem vistas nas páginas da imprensa ilustrada, as imagens de enfermeiras-parteiras formadas pela Pró-Matre remetiam os leitores às enfermeiras formadas pela Cruz Vermelha Brasileira, em função da apropriação do véu e do símbolo da cruz em seus uniformes ${ }^{8}$. Cabe ressaltar que o símbolo da cruz era na cor verde, em virtude de um grupo de mulheres daquela instituição ser reconhecido como as Damas da Cruz Verde.

Na década de 1920, houve diversos marcos para a enfermagem, ressaltando-se a realização do Congresso Nacional dos Práticos (1922). Neste, Ethel Parsons fez-se presente ao participar de duas arenas de debate: enfermeiras versus médicos e parteiras versus médicos ${ }^{31}$. A última arena visava resolver dois tipos de problemas encontrados pelos médicos. O primeiro consistia em eliminar as parteiras leigas e o segundo visava combater a insubordinação das parteiras diplomadas, que não se submetiam aos médicos e às determinações legais vigentes. Portanto, o que se encontrava em jogo era a exclusividade dos médicos em exercerem a arte de partejar de forma exclusiva. Para Parsons, isto implicava a defesa em prol das enfermeiras de saúde pública e, logo, colocar em prática os objetivos de sua missão: implantar a enfermagem moderna e enunciar a imagem pública da enfermeira ${ }^{32}$

Em um estudo sobre as publicidades das instituições de saúde articuladas à imagem pública da enfermeira no Distrito Federal, no período de 1917-1931, foi possível identificar enfermeiras com os atributos de marcadores identitários da profissão: véu, gorro e touca. As instituições que mais se destacaram foram o Hospital Internacional of Brazil e a Casa de Saúde Maternidade Dr. Pedro Ernesto, cada uma com 14\%, e, com menor percentual (3\%), o Sanatório Guanabara, Hospital Evangélico e a Casa de Saúde Dr. Oliveira Motta. As imagens das enfermeiras retratadas por estas instituições foram assim apresentadas: 100 com véu, 60 com gorro, quatro com touca e 111 articuladas ao símbolo da cruz, sem distinção de cor².

Os dados evidenciaram a predominância do uso do véu com o símbolo da cruz, o que representava marcadores institucionais das enfermeiras formadas pela Cruz Vermelha Brasileira, bem como de outras instituições que seguiam sua liderança. Esses dois marcadores não eram ostentados pelas enfermeiras formadas pela Escola de Enfermeiras do Departamento Nacional de Saúde Pública.

Estudo apontou que a Escola de Enfermeiras do Departamento Nacional de Saúde Pública, desde a sua criação até a formação da primeira turma (1922-1925), realizou vários investimentos em publicidades ${ }^{1}$, destacando-se a imagem apresentada na Figura 3. Esta se destinava à propaganda para a formação da enfermeira, quando o profissional que editou a matéria ilustrou-a com a imagem de enfermeira com véu, o que não atendia ao atributo da instituição pautada (imagem à esquerda). A outra imagem, peça da mesma instituição, no ano de formação de sua primeira turma, apresentava os requisitos para as moças que se candidatassem à profissão com garantia de emprego após a formatura.
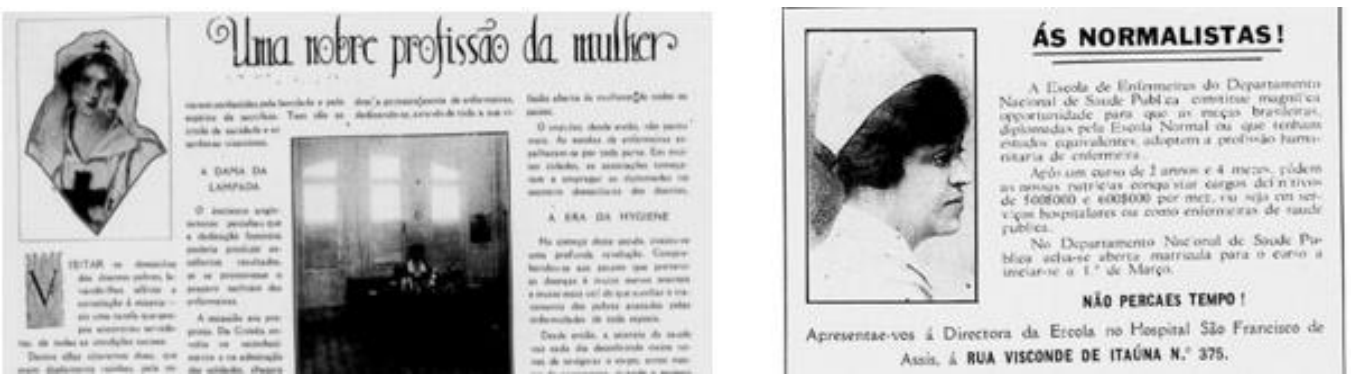

FIGURA 3: Propagandas - Revista da Semana, Rio de Janeiro, 1925.

As propagandas produzem certos sentidos. Por um lado, como uma das maneiras de se fazer ver e crer, como estratégia de demarcação no campo de luta, pela concorrência com a Escola Prática de Enfermeiras da Cruz Vermelha Brasileira; e, por outro lado, mostrar a garantia de emprego da enfermeira de saúde pública, estabelecendo a crença para a formação profissional no campo da saúde.

Outro estudo, com peças publicitárias de remédios, evidenciou a presença de representações de enfermeiras pelos mesmos marcadores identitários. Com eles, o estudo ressaltou (1916-1931) frequências diversas, com predominância do véu e do símbolo da cruz. Essa investigação possibilitou a assertiva de que a liderança na enunciação da imagem era, de fato, da Cruz Vermelha ${ }^{33}$. Isto se deve pela credibilidade das formadas pela instituição, pois a 
veiculação da publicidade de remédios, com a representação delas, reforçava marcas simbólicas da cultura visual que se tinha sobre a credibilidade e distinção da instituição.

Estudo sobre as enfermeiras do Departamento Nacional de Saúde Pública, durante a Reforma Sanitária, identificou crise na formação dessas enfermeiras no final da década de $1920^{9}$. À primeira vista, a assertiva parece ser refutável, mediante a publicação de uma matéria veiculada na Revista da Semana intitulada As missionárias da caridade, datada de $1929^{34}$. Contudo, ao ser analisada e discutida com base nas circunstâncias e resultados de outro estudo ${ }^{35}$, a publicação tratava, de fato, de estratégia para motivar candidatas, considerando o desinteresse das enfermeiras de saúde pública, desmotivadas pelo excesso de trabalho e pela disciplina rígida atribuída à formação.

Outro estudo apontou a fragilidade da liderança pela Escola de Enfermeiras do Departamento Nacional de Saúde Pública, pondo em evidência que as comemorações do Dia da Enfermeira, na década de 1920, eram disputadas nas páginas das revistas pela Cruz Vermelha Brasileira ${ }^{7}$. A documentação examinada desvela a disputa das duas instituições de ensino na imprensa ilustrada, inclusive na diagramação de algumas páginas, na modalidade de mosaico, a ritualista realizada por ambas, quando a Cruz Vermelha Brasileira liderava, quantitativamente, nas imagens publicadas.

O presente debate historiográfico pode até ser refutado, mas, por meio das fontes históricas, não se pode negar a liderança da enunciação da imagem pública da enfermeira pela Escola Prática de Enfermeiras da Cruz Vermelha Brasileira àquela época. Pensar nessa perspectiva é considerar que o fato histórico é sangue coagulado que volta a escorrer impulsionado por fatos; ferida que se faz presente pela descoberta de outros documentos no debate historiográfico ${ }^{36}$. Isto implica a ideia de que ele se faz necessário, considerando que outras versões e interpretações são possíveis.

Entender como ocorreu a construção da imagem pública da enfermeira, pelo pensamento histórico, é investir e ampliar horizontes pelos conhecimentos adquiridos e reproduzidos ao articular posições e suposições que dela resultaram.

A formação de enfermeiras, por meio de escolas/cursos, deu-se também na enunciação da imagem pública da enfermeira pela concorrência apontada pelos estudos em debate neste artigo. Nesse sentido, isto pode ser entendido pela expressão a duras penas ${ }^{37}$, significando o sacrifício da labuta do trabalho, sob rígida disciplina e ensinamento para se profissionalizar. Lutaram entre si e excluíram os homens, bem como há indícios em outros estudos que, direta e/ou indiretamente, ratificam o dito ${ }^{38,39}$. Assim, pode estar certa a explicação do desinteresse da atual Escola de Enfermagem Alfredo Pinto na concorrência à época, pois suas estratégias na formação de profissionais visavam ambos os sexos, o que a fez atravessar dois séculos na formação de enfermeiros(as).

\section{CONCLUSÃO}

O debate historiográfico buscou elucidar as tramas com base nas imagens veiculadas em páginas das duas revistas que publicitavam o imagético da enfermeira e apoio de estudos sobre o tema. $O$ véu associado ao símbolo da cruz versus a touca disputou a liderança da enunciação da imagem pública da enfermeira nas páginas da imprensa ilustrada. Nessa luta, ganhar não significou necessariamente perder, e vice-versa.

Por um lado, a Escola Prática de Enfermeiras da Cruz Vermelha Brasileira enunciou a imagem nas duas décadas do século XX. Por outro lado, no início da década de 1930, ocorreu a reação, de forma política-educacional, da Escola de Enfermeiras Donna Anna Nery, na gestão do presidente da República Getúlio Vargas, tornando-se a instituição oficial padrão para o ensino da profissão no Brasil.

Por último, o debate historiográfico da enunciação da imagem pública da enfermeira apontou para a disputa de duas instituições internacionais - Fundação Rockefeller e Cruz Vermelha Internacional —, o que merece investimento intelectual no campo das relações internacionais para a construção da trajetória da enfermagem.

\section{REFERÊNCIAS}

1. Albuquerque Júnior DM. História - a arte de inventar o passado. Bauru (SP): EDUSC; 2007.

2. Ayres LFA, Amorim W, Porto F, Luchesi LB. As enfermeiras visitadoras da cruz vermelha brasileira e do departamento nacional de saúde pública no início do século XX. In: Porto F, Amorim W, organizadores. História da Enfermagem: identidade, profissionalização e símbolos. São Caetano (SP): Yendis; 2013. p. 217-79.

3. Baptista SS, Barreira IA. Condições de surgimento das escolas de enfermagem brasileiras (1890 - 1960). Rev. Alternativa de Enfermagem. 1997; 1(2):4-17.

4. Almeida Filho AJ. The historical research: theory, methodology and historiography. Hist. enferm. Rev. eletrônica [HERE] [internet], 2016 [cited 2018 Dec 10]. 7(2): 381-2. Available from: http://here.abennacional.org.br/here/2a01a.pdf.

5. Barreira IA. Os primórdios da enfermagem moderna no Brasil. Esc. Anna Nery Rev. Enferm. 1997. 1(1): 161-84. 
6. Carvalho AC. Associação Brasileira de Enfermagem - (1926-1976): documentário. Rev. bras. enferm. (Online) [Scielo-Scientific Electronic Library Online] [internet], 2002 [cited 2018 Dec 10]. 55(3): 249-63. DOI: http://dx.doi.org/10.1590/S003471672002000300003

7. Santos LAC, Faria LA. A duras penas: estratégias, conquistas e desafios da enfermagem em escala mundial. In: Santos LAC, Faria LA, organizadores. Saúde e história. São Paulo: Aderaldo e Rothschild; 2010. p.187-208.

8. Church OM. The emergence of training programmes for asylum nursing at the turno of the centry. Advance in nursing Science. 1985; 7(2): 33-46.

9. Coury A. Fatos e fotos da enfermeira da cruz vermelha brasileira no enfrentamento da gripe espanhola (1918) [dissertação de mestrado]. Rio de Janeiro: Universidade Federal do Estado do Rio de Janeiro; 2010.

10. Deslandes AKM. Cuidado e enfermeiras na revista da semana no âmbito da reforma sanitária [dissertação de mestrado]. Rio de Janeiro: Universidade Federal do Estado do Rio de Janeiro; 2012.

11. Dock LL, Nutting AM. The history of nursing. New York: GP Putnam's Sons; 1935.

12. Duque SJT. As escolas de enfermeiras da Cruz Vermelha Brasileira e a profissionalização da enfermagem (Rio de Janeiro, 18901923) [dissertação de mestrado]. Vassouras (RJ): Universidade Severino Sombra; 2012.

13. Faria L. Health educators and public health nurses: professional identities in the making. Cadernos Pagu [Scielo-Scientific Electronic Library Online] [internet], 2006 [cited 2018 Dec 12]. 27(2): 173-212. DOI: http://dx.doi.org/10.1590/S010483332006000200008.

14. Boschma G. The gender specific role of male nurses in Dutch asylums: 1890-1910. Int. Hist. Nurs. J. [internet], 1999 [cited 2018 Dec 14]. 4(3): 13-9. Available from: https://www.ncbi.nlm.nih.gov/pubmed/11623866

15. Fonseca EFR. A imagem pública da enfermeira-parteira do hospital maternidade Pró-Matre do Rio de Janeiro no período de 1928-1931: (des)construção de uma identidade profissional [dissertação de mestrado]. Rio de Janeiro: Universidade Federal do Estado do Rio de Janeiro; 2011.

16. Morais FTN, Amorim W. Os congressos médicos latino americanos e a enfermagem (1904-1907). In: Porto F, Amorim W organizadores. História da Enfermagem - identidade, profissionalização e símbolos. Bauru (SP): Yendis; 2013. p.87-110.

17. Mott M, Oguisso T. Discutindo os primórdios do ensino de enfermagem no Brasil: o curso de Enfermeiras da Policlínica de Botafogo (1917-1920). Rev. Paul. Enferm. 2003; 22(1): 82-91.

18. Mott ML, Tsunechiro MA. The brazilian red cross training schools for nurses and the origins of professional nursing in Brazil. Rev. bras. enferm. (Online) [Scielo-Scientific Electronic Library Online] [internet], 2002 [cited 2018 Dec 22]. 55(5): 592-9. DOI: http://dx.doi.org/10.5935/0034-7167.20020079.

19. Motion Picture Magazine [site de internet]. [cited 2018 Dec 20]. Available from: https://br.pinterest.com/pin/334462709814772345/.

20. Nascimento SA. "O Dia da Enfermeira" nas páginas da revista da semana (1929-1930): Anna Nery e os lucros simbólicos. [dissertação de mestrado]. Rio de Janeiro: Universidade Federal do Estado do Rio de Janeiro; 2013.

21. Neto M. A produção da crença na imagem da enfermeira da cruz vermelha brasileira no período da primeira guerra mundial (1917-1918) [dissertação de mestrado]. Rio de Janeiro: Universidade Federal do Estado do Rio de Janeiro; 2011.

22. Oguisso T, Dutra VO, Souza Campos PF. Cruz Vermelha Brasileira filial do Estado de São Paulo: formação em tempos e paz. São Paulo: Manole; 2009.

23. Pereira Neto A. Palavras, intenções e gestos: os interesses profissionais da elite medica: congresso nacional dos práticos (1922). Juiz de Fora (MG): Clio; 2002.

24. Porto F. Enfermagem: Cruz Vermelha e Anna Nery [relatório de pós-doutoramento]. São Paulo: Universidade de São Paulo; 2009.

25. Porto F. Os ritos institucionais e a imagem pública da enfermeira brasileira na imprensa ilustrada: o poder simbólico no click fotográfico (1919-1925) [tese de doutorado]. Rio de Janeiro: Universidade Federal do Rio de Janeiro; 2008.

26. Porto F. Nursing history in Brazil. Cultura de los Cuidados [Revista de Enfermería y Humanidades] [internet], 2009 [cited 2018 Dec 10]. 13(2): 7-8. DOI: https://doi.org/10.14198/cuid.2009.26.01

27. Porto F, Amorim WM. Escuelas de enfermería y cursos en la historia de la profesión en Brasil (1890-1922). Cultura de los Cuidados [Revista de Enfermería y Humanidades] [internet], 2010 [cited 2018 Dec 20]. 14(1): 40-45. DOI: https://doi.org/10.7184/cuid.2010.27.05

28. Porto F, Neto M, Goulart SM. Pieta: the image representation of care. Cultura de los cuidados [Revista de Enfermería y Humanidades] [internet], 2013 [cited 2018 Dec 20]. 17(3): 13-21. DOI: https://doi.org/10.7184/cuid.2013.37.02

29. Porto F, Neto M. Nurses in the brazilian illustrated press (1890-1925): signature imagery. Patrimônio e Memória [Patrimônio e Memória UNESP] [internet], 2014 [cited 2018 Dec 21]. 10(1): 199-221. Available from: http://pem.assis.unesp.br/index.php/pem/article/view/421/737

30. Revista da semana. As missionárias da caridade. In: Revista da Semana (Rio de Janeiro) 1929; 14:16.

31. Revista da semana. Pelo mundo a fora. The greatest mother in the world In: Revista da Semana (Rio de Janeiro) 1918; $20: 27$.

32. Santos TCF, Barreira IA. O poder simbólico da enfermagem norte-americana no ensino da capital do Brasil (1928-1938). Rio de Janeiro: Escola Anna Nery; 2002.

33. Sauthier J, Barreira IA. As enfermeiras norte-americanas e o ensino da enfermagem na capital do Brasil: 1921-1931. Rio de Janeiro: Escola Anna Nery; 1999.

34. Silva KFT, Villela DO, Risi L, Rocha JA, Porto F. The image of the female nurse in medication advertisements in Brazil (1916 1931). Rev. Enf. Ref. [Scielo-Scientific Electronic Library Online] [internet], 2015 [cited 2018 Dec 20]. 4(7): 123-28. DOI: http://dx.doi.org/10.12707/RIV15053 
35. Veraldo TX. Publicidade das instituições de saúde e a imagem pública da enfermeira brasileira nas páginas da Fon-Fon (19171930) [dissertação de mestrado]. Rio de Janeiro: Universidade Federal do Estado do Rio de Janeiro; 2013.

36. Santos GF. O livro do enfermeiro e da enfermeira para uso das pessoas que se destinam à profissão de enfermeiro e das pessoas que cuidam de doentes. Rio de Janeiro: Tip. Jornal do Comércio, 1928.

37. Moreira A. The professional school of nurses (1906). Rev. pesqui. cuid. fundam. (Online) 2010 [cited 2018 Dec 28]. 2(3): 118183. Available from: http://www.redalyc.org/articulo.oa?id=505750832024

38. Neto M, Nassar PR, Freitas TM, Porto F. Newborn care: hygiene and clothing, in the nineteenth century. Rev. enferm. UERJ. [internet], 2013 [cited 2018 Dec 28]. 21(2): 192-6. Available from: https://www.epublicacoes.uerj.br/index.php/enfermagemuerj/article/view/7104/5016

39. Fonseca EFR, Porto F. Nurse-midwives and their uniform: object representations and evidence in the construction of professional identity. Rev. enferm. UERJ. [internet], 2011 [cited 2018 Dec 28]. 19(3): 432-7. Available from: http://www.facenf.uerj.br/v19n3/v19n3a16.pdf 